\title{
Revisão sobre modelagem matemática na logística humanitária
}

\author{
Sergio Argollo da Costa1, Renata Albergaria de Mello Bandeira², \\ Vania Barcellos Gouvêa Campos ${ }^{3}$, Adriano Fontainhas de Paula Bandeira ${ }^{4}$
}

\begin{abstract}
Resumo: Este trabalho apresenta uma revisão de artigos científicos que propõem uma modelagem matemática para atuação na logística humanitária. A partir desta revisão, foram levantados os principais métodos utilizados dentro de três etapas da cadeia de suprimento humanitária: Aquisição, Armazenagem e Transporte/Distribuição. Também foram identificadas as principais variáveis de decisão utilizadas nos modelos, sendo enfatizadas as particularidades de tais variáveis no contexto da logística humanitária. Desta forma, foi possível perceber as dificuldades de utilização de modelos em operações de resposta a desastres. Portanto, são discutidos aspectos associados às características que cercam o desenvolvimento e a funcionalidade de cada modelo, no que diz respeito às possibilidades de maior aderência do modelo matemático às reais condições operacionais da ação de resposta humanitária.
\end{abstract}

Palavras-chave: Logística humanitária. Desastres naturais. Modelos matemáticos.

Abstract: The paper reviews scientific papers published in the literature that propose mathematical models to be applied in the field of humanitarian logistics. From this review, the main methods used in three stages of the humanitarian supply chain (Acquisition, Storage and Transportation/Distribution) were assessed. Moreover, the main decision variables used in these models were identified, emphasizing the peculiarities of such variables in the context of humanitarian logistics. Therefore, it was possible to understand the difficulties in applying mathematical models in disaster response operations. For that reason, the paper discusses aspects related to the development and the functionality of such mathematical models, regarding the possibilities of greater adherence of mathematical models to the actual operating conditions of humanitarian response operations.

Keywords: Humanitarian logistics. Natural disasters. Mathematical modeling.

\section{INTRODUÇÃO}

Desastres são definidos como eventos súbitos e calamitosos que interrompem as atividades de uma sociedade ou comunidade, causando perdas humanas, materiais, econômicas ou ambientais que excedem a capacidade de recuperação da sociedade atingida usando apenas seus próprios recursos (Natarajarathinam et al., 2009). De acordo com IFRC (2000), desastres são classificados segundo: (i) a causa do evento; (ii) a velocidade de início (súbito ou lento); e (ii) se são devido a "atos da natureza" ou "atos de seres humanos". Essa classificação se faz necessária para que sejam definidas as medidas de preparação, de resposta e de redução de riscos, bem como os especialistas e órgãos envolvidos em determinado desastre.

\footnotetext{
1 Universidade Estácio de Sá (sergioargollo@yahoo.com.br).

2 Instituto Militar de Engenharia (re.albergaria@gmail.com).

3 Instituto Militar de Engenharia (vania@ime.eb.br).

4 Instituto Militar De Engenharia (adrianopfb@gmail.com).
}

Manuscrito recebido em 25/09/2013 e aprovado para publicação em 17/06/2014. Este artigo é parte de TRANSPORTES v. 22, n. 2, 2014. ISSN: 2237-1346 (online).

DOI: http://dx.doi.orq/10.14295/transportes.v22i2.732.
O objeto de estudo deste artigo são desastres naturais, ou seja, eventos oriundos de fenômenos e desequilíbrios da natureza que ocorrem com ou sem aviso prévio, acarretando perdas humanas e materiais. Este tipo de desastre geralmente obtém maior atenção da mídia, o que, muitas vezes, leva a um maior financiamento. Entretanto, tais desastres apresentam, cada vez mais, impactos maiores sobre comunidades e nações por todo o mundo e previsões confirmam que esta tendência deve continuar (Guha-Sapir, 2013). Nas últimas três décadas, a ocorrência de desastres naturais aumentou de 50 para 400 por ano (Kovács e Spens, 2007). Para os próximos 50 anos, é esperado que esta taxa se torne cinco vezes maior (Thomas e Kopczack, 2005).

O cenário instável e aleatório imposto por um desastre natural gera uma tarefa de difícil solução no contexto de alívio humanitário, exigindo a presença de uma gestão adequada para a melhoria do planejamento da resposta. Castro (1999) e Van Wassenhove (2006) consideram o gerenciamento de desastres em quatro fases: Prevenção, Preparação, Resposta e Reconstru- 
ção. Kovács e Spens (2007) distinguem as operações de gestão de desastres pelo tempo: antes de um desastre (prevenção e preparação), imediatamente após um desastre (resposta) e, na sequência de um desastre (reconstrução). O presente artigo se foca nas operações de resposta a desastres naturais. Nestas ações, atrasos na entrega de suprimentos ou na prestação de ajuda podem custar vidas. Portanto, a eficiência logística é um aspecto crítico para o sucesso de uma ação humanitária deste tipo por garantir o fluxo de bens e serviços em uma cadeia de suprimentos complexa.

Neste contexto, torna-se essencial a busca, por meio da Logística Humanitária, de um procedimento eficiente para planejar, implantar e controlar estoques de suprimentos, bem como acompanhar o fluxo de informações, desde a origem até o consumo, com o propósito de atender aos requisitos do beneficiário final (Thomas e Mizushima, 2005; Thomas e Kopczak, 2005). A logística desempenha um papel fundamental nas operações de resposta a desastres, sendo crucial para a eficácia e capacidade de resposta (Thomas, 2003). No entanto, na logística humanitária, as decisões costumam ser tomadas com urgência e com base na experiência e na intuição dos profissionais envolvidos. O processo decisório durante este tipo de operação é complexo, uma vez que atributos importantes do problema são incertos e as necessidades mudam rapidamente, além do pouco tempo disponível e dificuldade em obter informações necessárias para a tomada de decisão (Beamon e Balcik, 2005). Considerando esse contexto, foram pesquisados, na literatura, modelos matemáticos, que, com base em técnicas de Pesquisa Operacional, podem ser aplicados na solução de desafios da logística humanitária em operações de resposta a desastres naturais e, assim, auxiliar na tomada de decisão. Acredita-se que tais modelos podem ser adaptados para apoiar o processo logístico em operações deste tipo no Brasil. Contudo, convém destacar a limitada quantidade de trabalhos publicados sobre redes de distribuição de resposta rápida para cadeias de suprimentos humanitárias (Beamon e Balcik, 2005; Perez et al., 2010).

Portanto, o presente trabalho apresenta uma revisão de artigos científicos que propõem uma modelagem matemática que contribui para a melhoria do desempenho logístico nas respostas humanitárias a desastres naturais. Os traba- lhos selecionados foram agrupados de forma a permitir uma melhor compreensão de suas aplicações e das contribuições às etapas da cadeia de suprimentos humanitária que compõem escopo deste estudo (isto é, aquisição e captação; armazenagem e movimentação; distribuição e transporte). Em seguida, estes modelos matemáticos são analisados e enquadrados segundo seus objetivos, a abordagem logística, o momento de aplicação e variáveis de decisão. Posteriormente, são identificadas e discutidas as técnicas de Pesquisa Operacional aplicadas pelos autores na modelagem de suas propostas. Enfim, é apresentada uma análise da possibilidade do uso das soluções propostas pelos artigos para a correção dos problemas observados em respostas a desastres, avaliando as condições em que possam contribuir para que as operações logísticas atinjam melhores resultados.

\section{SELEÇÃO DOS ARTIGOS PARA ESTUDO}

Para elaboração deste trabalho, buscaramse artigos da literatura de logística humanitária, que propõem modelos matemáticos que se baseiam em técnicas de Pesquisa Operacional e podem ser aplicados como ferramentas de apoio à tomada de decisões relacionadas à cadeia de suprimentos humanitária em operações de resposta a desastres. Contudo, o presente estudo foca apenas nas decisões relacionadas aos seguintes processos logísticos:

- Aquisição/captação: atividades relativas à preparação, planejamento e avaliação da cadeia de suprimentos;

- Armazenagem e movimentação: referente às questões operacionais de armazenamento e movimentação de materiais para abastecimento da cadeia de suprimentos e planejamento de estoques;

- Transporte e distribuição: associado à entrega do produto ao usuário final ou a um ponto para redespacho.

Thomas (2003) propõe um modelo de sete etapas que representa os processos logísticos da cadeia de suprimentos humanitária. A Figura 1 ilustra o modelo proposto por Thomas (2003) e destaca os processos logísticos que são objetos de estudo deste trabalho. 


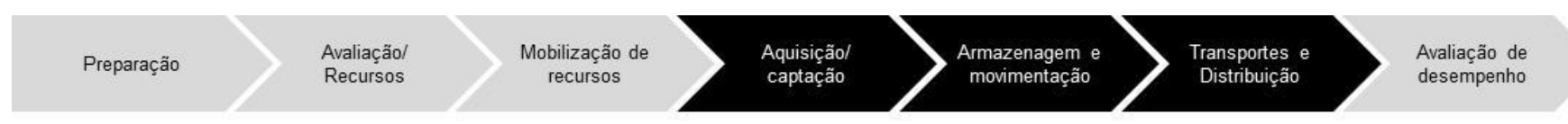

\section{Escopo do estudo}

Figura 1 - Problemas na cadeia de suprimentos humanitária Fonte: Adaptado de Thomas (2003)

Para o desenvolvimento deste estudo, foram levantados somente artigos indexados em bases de dados bibliográficas. Inicialmente, foram definidas as palavras-chave "Humanitarian" e "Logistics", que foram combinadas da seguinte forma: ("Humanitarian" E "Logistics"). O processo de coleta dos artigos foi realizado em três bases ISI Web of Knowledge, Scopus e Science Direct, considerando-se as publicações em periódicos e em anais de congresso entre 2001 e 2012. Ainda, nas bases de dados, foi realizada a última restrição, selecionando os campos "Pesquisa Operacional e Ciências Gerenciais" e "Transportes" como "Áreas de Pesquisa". Neste momento, foram levantados quarenta e quatro artigos.

Após esta coletânea inicial dos artigos, fo- ram separados aqueles que propõem modelos matemáticos para tratar das atividades logísticas inerentes ao escopo da cadeia de suprimentos em estudo (aquisição/captação; armazenagem e movimentação; transporte e distribuição). Assim, dos quarenta e quatro artigos inicialmente selecionados, vinte e cinco são a base da revisão apresentada neste trabalho.

A análise dos objetivos propostos pelos autores permitiu o enquadramento dos artigos estudados às etapas da cadeia de suprimento humanitária definidas como escopo deste trabalho, para uma melhor compreensão da abordagem de cada modelo na busca de melhorias no desempenho de atividades logísticas. A Tabela 1 apresenta o resultado deste primeiro enquadramento.

Tabela 1 - Enquadramento dos artigos às etapas da cadeia de suprimentos

\begin{tabular}{|c|l|}
\hline Etapas do processo logístico & Autores \\
\hline AQUISIÇÃO/CAPACITAÇÃO & $\begin{array}{l}\text { Nagurney et al. (2012); Nagurney et al. (2010); Salmeron e Apte (2010); } \\
\text { Clark e Culkin (2007); Beamon e Balcik (2005); Rawls e Turnquist } \\
\text { (2009); Mete e Zabinsky (2009); Yushimito e Ukkusuri (2007); Beamon e } \\
\text { Balcik (2008) }\end{array}$ \\
\hline $\begin{array}{c}\text { ARMAZENAGEM E } \\
\text { MOVIMENTAÇÃO }\end{array}$ & $\begin{array}{l}\text { Wyk et al. (2011); Emmet e Lodree (2011); Ozbay e Ozguven (2007); } \\
\text { Beamon e Kotleba (2006); Jaller e Holguín-Veras (2011); Günnec e } \\
\text { Salman (2007); Yi e Özdamar (2007); Huang et al. (2012); }\end{array}$ \\
\hline $\begin{array}{l}\text { Falasca et al. (2009) } \\
\text { DISTRIBUIÇÃO }\end{array}$ & $\begin{array}{l}\text { Lin et al. (2010); Perez et al. (2010); Lin et al. (2009); Campbell et al. } \\
\text { (2008); Barbarosoglu e Arda (2004); Özdamar et al. (2004) }\end{array}$ \\
\hline
\end{tabular}

\section{ANÁLISE DOS ARTIGOS SELECIONADOS SEGUNDO A ETAPA DA CADEIA HUMANITÁRIA E ABORDAGEM LOGÍSTICA}

Conforme apresentados na Tabela 1, os artigos foram agrupados para cada etapa da cadeia de suprimentos humanitários com o intuito de identificar suas aplicações e a abordagem para melhoria das atividades logísticas inerentes à etapa. A seguir, os resultados desta análise são apresentados para cada etapa da cadeia humanitária analisada nesta pesquisa. 


\subsection{Aquisição/Captação}

Para o planejamento das atividades relacionadas à aquisição e captação de suprimentos (estrutura e operação), foram identificadas nos artigos duas abordagens: a primeira relacionada à otimização da rede de suprimentos; e a segunda tratando do pré-posicionamento de produtos, que são apresentadas a seguir, com a identificação dos respectivos modelos.

\subsubsection{Otimização da rede de suprimentos}

As propostas de Nagurney et al. (2012), Nagurney et al. (2010), Salmeron e Apte (2010), e Clark e Culkin (2007) são modelos multiprodutos que visam o desenho ou o redesenho da rede da cadeia de suprimentos através da minimização do custo total e da demanda não atendida. Em Nagurney et al.(2012), a solução indica o investimento ótimo na capacidade da rede (fabricação, armazenagem e transporte/expedição), enquanto em Nagurney et al. (2010) a perspectiva do desenho da rede é flexibilizada por considerar a possibilidade de terceirização na busca de melhores alternativas na relação capacidade-custo. Salmeron e Apte (2010) utilizam o pré-posicionamento de recursos estratégicos para a otimização da rede de suprimentos de ajuda humanitária através de um modelo estocástico para aplicação em áreas geográficas caracterizadas por desastres cíclicos. O modelo de rede de Clark e Culkin (2007) estuda possíveis cenários (incluindo operações de transbordo), fornecendo informações sobre a quantidade de itens transportados e o número de veículos necessários para fazê-lo.

Com a mesma finalidade de redesenho da cadeia de suprimentos, Beamon e Balcik (2005), diferentemente dos demais, focam no tempo de resposta ao desastre como o principal objetivo no cenário de ações humanitárias, buscando minimizar a soma dos tempos de resposta através do conhecimento do risco das áreas possivelmente afetadas. Agregam ao resultado a melhor estratégia de distribuição considerando, para este fim, o trade-off entre a armazenagem ou o envio direto do fornecedor. Os autores simplificam o modelo trabalhando com apenas um modo de transportes, instalações idênticas e desconsiderando eventuais danos causados pelo desastre às instalações e à infraestrutura de transportes.

Nagurney et al. (2012) e (2010), e Salmeron e Apte (2010) valorizam os objetivos do contexto humanitário através do estabelecimento de componentes de custos que penalizam o não cumprimento da demanda, e que compõem o custo total a ser minimizado. Os primeiros também estabelecem penalidades para excedentes de estoque, enquanto Salmeron e Apte (2010) propõem a conversão da quantidade de produto não entregue em perdas de vidas. A aplicação de pesos às parcelas da função objetivo é a alternativa adotada por Clark e Culkin (2007), permitindo ao decisor estabelecer quais deverão ser minimizadas com maior ênfase.

\subsubsection{Pré-posicionamento de produtos}

Os modelos identificados que abordam especificamente o pré-posicionamento de produtos, tratam de três objetivos: custos, tempo e nível de atendimento; sendo que apenas o trabalho de Mete e Zabinsky (2009) consideram simultaneamente estes três fatores através da minimização dos custos operacionais dos depósitos, do tempo de transporte e da penalização pelo não atendimento da demanda. O modelo proposto por estes autores (2009) considera suprimentos médicos em diversos cenários de ocorrência de desastre para as fases de preparação e de resposta, sendo parte de um programa da Universidade de Washington voltado para a costa do Pacífico (PARVAC).

O modelo de Rawls e Turnquist (2009) avalia os possíveis cenários pós-desastre, buscando minimizar o custo da operação a partir de decisões referente à localização de instalações, aos níveis de estoque para suprimentos de emergência e à distribuição para múltiplos pontos de demanda. Yushimito e Ukkusuri (2007) tratam a questão de pré-posicionamento de estoques com base na confiabilidade da rede de transporte terrestre, buscando maximizar a certeza da entrega ao ponto de demanda. Por sua vez, Beamon e Balcik (2008) tratam da questão dos centros de distribuição na rede de ajuda humanitária e da quantidade de suprimentos de socorro a serem estocados em escala global, através da discussão dos impactos da decisão pelo pré-posicionamento ou não de itens críticos sobre o tempo de resposta e o atendimento à demanda (principalmente a influência da atividade de aquisição).

Os trabalhos consideram os eventuais danos causados a infraestrutura pela ocorrência do desastre, adequando suas soluções ao contexto humanitário através do estabelecimento de com- 
ponentes de custos a serem minimizados, funcionando como penalidades para o não atendimento à demanda ou para a sobra de itens em estoque.

\subsection{Armazenagem e Movimentação}

Lidando com temas associados ao controle e gestão de materiais, os artigos enquadrados nesta etapa seguem as seguintes diretrizes de desenvolvimento: planejamento de estoques, localização de instalações e alocação de pessoal.

\subsubsection{Planejamento de estoques}

Dos modelos voltados ao planejamento dos estoques, as propostas de Wyk et al.(2011), de Emmett e Lodree (2011), Ozbay e Ozguven (2007) e de Beamon e Kotleba (2006) tratam da definição de políticas para a manutenção dos estoques em instalações pré-definidas, visando agilizar a distribuição de itens de ajuda humanitária às vítimas de desastres naturais, estabelecendo como objetivos a minimização dos custos e maximização do atendimento. Tanto Wyk et al. (2011) quanto Beamon e Kotleba (2006) penalizam o não atendimento através de um componente de custo associado, que compõe a função objetivo dos modelos juntamente com os custos operacionais de se manter o inventário. Sobre o custo do não atendimento, Wyk et al.(2011) o identificam como "custo de escassez", e aplicam ao cálculo uma estimativa do valor monetário da vida usando como base o custo da lesão fatal por pessoa determinada pelo Departamento Nacional de Transporte na África do Sul (De Beer e Van Niekerk, 2004). Ozbay e Ozguven (2007) apresentam um modelo complexo, propondo uma aplicação do Modelo Húngaro de Controle de Estoques de Prékopa (2006), para o equilíbrio entre a expedição e o consumo das regiões afetadas a um mínimo custo.

A proposta de Emmett e Lodree (2011), embora enquadrada no contexto da Logística Humanitária, trata de uma avaliação da decisão do varejista quanto ao melhor momento para a aquisição de produtos de emergência para cenários de desastre, mediante a probabilidade de sua ocorrência. $\mathrm{O}$ modelo propõe ao varejista uma solução baseada no mínimo custo e no máximo lucro, com características mais comerciais do que humanitárias, podendo ser utilizada neste último contexto se aplicado de forma similar à aquisição de itens críticos por agências de ajuda humanitária para pré-posicionamento de estoques, na aproximação da ocorrência de desastres naturais com perfil de frequência cíclico.

\subsubsection{Localização de instalações}

As propostas apresentadas para esta função da logística se deparam com as questões clássicas para a solução do problema de localização de instalações. As tentativas de diferenciação das propostas dos autores estão associadas à modelagem incluindo outra função da logística. Yi e Özdamar (2007) e Huang et al.(2012) incluem a questão da roteirização; enquanto Jaller e Houguín-Veras (2011) adicionam uma política de estoque a sua proposta. Günnec e Salman (2007) propõem uma revisão pós-desastre baseada nas consequências da ocorrência do evento às instalações determinadas previamente.

A abordagem em dois estágios proposta por Günnec e Salman (2007) utiliza uma alternativa logística que oferece flexibilidade à tomada de decisão, permitindo a transformação de localizações específicas de transbordo de produtos para operarem como depósitos, diante dos danos à infraestrutura após o desastre natural. Da mesma forma que Clark e Culkin (2007) no tratamento da otimização da rede de suprimentos, os autores concedem ao decisor o estabelecimento de prioridades, associadas aos tempos de viagem e de espera, risco dos locais, atendimento à demanda e aos custos.

Jaller e Houguín-Veras (2011) aprofundam o tratamento direto à questão humanitária em seu modelo, agregando à função objetivo um componente relativo ao custo social, traduzido através do sofrimento humano provocado pela distância das vítimas aos pontos de distribuição de ajuda, e outra referente à privação ocasionada pelo congestionamento nestes locais. Os autores utilizam fatores de penalização para o não atendimento à demanda, mas tentam um método menos subjetivo para determiná-los, tratando aspectos reais relacionados às vítimas no cenário pós-desastre.

A utilização da roteirização integrada ao problema de localização com o objetivo de maximizar a taxa de serviço é a base dos trabalhos de Yi e Özdamar (2007) e Huang et al.(2012), preocupados em estabelecer um nível de equilíbrio ao serviço prestado às áreas atingidas pela máxima capacidade das instalações com o apoio do melhor roteiro, ou pelo uso racional da capacidade de transporte maximizando a satisfação 
mínima entre os pontos de demanda.

\subsubsection{Alocação de pessoal}

O trabalho de Falasca et al. (2009) é o único dentre os artigos estudados que trata da questão no campo do gerenciamento de voluntários em atividades de ajuda humanitária. A proposta apresenta dois objetivos: reduzir a falta de pessoal nas tarefas e o número de alocações de voluntários a tarefas que não tenham se disposto a executar (este aspecto, segundo os autores, gera desmotivação, com a consequente queda de eficiência). Assim, os autores apresentam a coordenação do trabalho de voluntários como um desafio da logística humanitária e a compara à do trabalhador remunerado através de uma adaptação da tabela de Sampson (2006). É proposto o uso do "The Sahana Volunteer Management Project", que é um módulo de um software livre para logística humanitária, desenvolvido pela Humanitarian FOSS Project@, que permite aos usuários coordenar as informações de contato, as competências, atribuições e disponibilidade de voluntários e equipes.

\subsection{Transporte e Distribuição}

A entrega efetiva às vítimas de desastres naturais torna a distribuição de produtos e a gestão dos transportes pontos-chave para uma bem sucedida ação humanitária. Neste sentido, os autores, que direcionaram seus trabalhos para esta etapa, procuram fazê-lo através da melhor escolha das rotas, ou da melhor coordenação destas atividades.

\subsubsection{Roteirização de entregas}

Os modelos, propostos pelos artigos estudados, voltados para o problema de roteirização no contexto humanitário tratam, de forma geral, das questões referentes ao tempo e ao atendimento à demanda, de maneira similar a aplicação para o contexto comercial. As adequações às condições operacionais da ação humanitária ocorrem em duas linhas: na primeira, determinam penalizações para o não atendimento e a sobra de estoque de ajuda humanitária. Nesta linha, Lin et al. (2009) e (2010) estabelecem prioridades baseadas na urgência da entrega dos itens críticos, em que a janela de tempo limita o prazo de envio a partir do qual o não cumprimento gera uma penalização, não claramente descrita pelos autores. Acrescentam um valor à questão humanitária através da tentativa desse- quilibrar o serviço prestado aos diversos demandantes. Em 2010, os autores apresentaram um trabalho com a aplicação da solução proposta em 2009 através da simulação de cenários de um terremoto no sul da Califórnia, realizando uma série de análises de sensibilidade e em que avaliam a influência de parâmetros como a localização de depósitos e o número de veículos disponíveis sobre o desempenho da roteirização em operação humanitária de resposta a desastre. Por sua vez, Barbarosoglu e Arda (2004) definem suas penalizações de forma subjetiva, em um modelo de dois estágios, considerando a multimodalidade e pontos de transferência ("crossdocking"), com seus respectivos custos.

A segunda linha é proposta por Perez et al.(2010), que avaliam a minimização dos custos compostos por uma parcela operacional e outra social; a mesma filosofia proposta em Jaller e Houlguín-Veras (2011) - este último também coautor do artigo em questão, em que se utiliza do sofrimento humano como um critério de decisão, embora reconheçam a dificuldade de estabelecer um custo para tal. Apesar disto, os autores propõem uma métrica para a privação das vítimas ao socorro baseada em abordagens aplicadas em transplantes de órgãos, políticas de saúde, indústria de seguros de vida e sistemas de triagem. O resultado é um modelo de solução complexa e de aplicação em pequenos eventos. O trabalho de Campbell et al. (2008), também classificado neste grupo, não propõe um modelo para aplicação, mas uma avaliação da mudança de indicadores e paradigmas no contexto humanitário através da discussão sobre o "trade-off" entre a simples e clássica meta de redução de custos, e a redução do tempo máximo e da soma dos tempos de entrega (considerados fatores importantes no ambiente de ações de ajuda humanitária), através da observação do número adicional de vítimas atendidas.

\subsubsection{Coordenação do fluxo de distribuição}

Özdamar et al.(2004) propõem um modelo para a coordenação do fluxo logístico de materiais associado à programação de transportes (modais e pontos múltiplos). O objetivo é minimizar a demanda total de produtos não atendida a partir da atualização em intervalos regulares de tempo das informações sobre demanda, suprimentos e disponibilidade de veículos. 
Enfim, a Tabela 2 sintetiza o enquadramento dos artigos analisados às etapas da cadeia de suprimentos de que tratam e à abordagem para melhoria de atividade logística inerente a etapa, permitindo uma visão resumida da distribuição dos objetivos dos modelos matemáticos estudados.

Tabela 2 - Enquadramento dos artigos estudados às etapas do Processo Logístico Humanitário

\begin{tabular}{|c|c|c|}
\hline Etapas da cadeia de suprimentos & Abordagem logística & Autores \\
\hline \multirow{2}{*}{ AQUISIÇÃO/CAPACITAÇÃO } & Otimização da rede de suprimentos & $\begin{array}{l}\text { Nagurney et al. (2012) } \\
\text { Nagurney et al. (2010) } \\
\text { Salmeron e Apte (2010) } \\
\text { Clark e Culkin (2007) } \\
\text { Beamon e Balcik (2005) }\end{array}$ \\
\hline & Pré-posicionamento de produtos & $\begin{array}{l}\text { Rawls e Turnquist (2009) } \\
\text { Mete e Zabinsky (2009) } \\
\text { Yushimito e Ukkusuri } \\
\text { (2007) } \\
\text { Beamon e Balcik (2008) }\end{array}$ \\
\hline \multirow{3}{*}{$\begin{array}{l}\text { ARMAZENAGEM E } \\
\text { MOVIMENTAÇÃO }\end{array}$} & Planejamento de estoques & $\begin{array}{l}\text { Wyk et al. (2011) } \\
\text { Emmet e Lodree (2011) } \\
\text { Ozbay e Ozguven (2007) } \\
\text { Beamon e Kotleba (2006) }\end{array}$ \\
\hline & Localização de instalações & $\begin{array}{l}\text { Jaller e Holguín-Veras } \\
(2011) \\
\text { Günnec e Salman (2007) } \\
\text { Yi e Özdamar (2007) } \\
\text { Huang et al. (2012) }\end{array}$ \\
\hline & Alocação de pessoal & Falasca et al. (2009) \\
\hline \multirow[t]{2}{*}{ TRANSPORTE E DISTRIBUIÇÃO } & Roteirização de entregas & $\begin{array}{l}\text { Lin et al. }(2010) \\
\text { Perez et al. }(2010) \\
\text { Lin } \text { et al. }(2009) \\
\text { Campbell } \text { et al. }(2008) \\
\text { Barbarosoglu e Arda (2004) }\end{array}$ \\
\hline & $\begin{array}{l}\text { Coordenação do fluxo de } \\
\text { distribuição }\end{array}$ & Özdamar et al. (2004) \\
\hline
\end{tabular}

\section{ANÁLISE DOS ARTIGOS \\ SELECIONADOS SEGUNDO O \\ MOMENTO DE APLICAÇÃO E AS \\ VARIÁVEIS DE DECISÃO DOS MODELOS PROPOSTOS}

O momento da aplicação e as variáveis de decisão utilizadas são características importantes que compõem a diretriz de desenvolvimento e a resposta esperada pelo modelo matemático proposto. No contexto humanitário, tais características determinam a estratégia e o foco na aplicação, associado à agilidade da ação com base na disponibilidade de material e na efetiva utilização dos recursos de transportes de forma a determinar o nível de serviço oferecido.

\subsection{Momento da aplicação}

Com relação ao momento da aplicação, foram identificados modelos que apresentam soluções direcionadas à preparação para a operação de resposta e outras à ação em si, após o evento.
As questões principais que dizem respeito à preparação e à resposta no ambiente da logística humanitária estão associadas à imprevisibilidade do cenário, ao tempo de resposta, variabilidade da demanda, à disponibilidade de informações e recursos - materiais e financeiros (Beamon e Balcik, 2005). Dentre os trabalhos analisados, há modelos para aplicação nas duas etapas, individualmente ou integradas. Estes últimos trabalham com modelos de dois estágios, buscando a melhoria dos resultados do estágio de resposta.

Long e Wood (1995) e McGuire (2001) consideram que a característica única de cada desastre é a principal causa para a dificuldade do planejamento durante os estágios iniciais da resposta humanitária, resultando na frequente falta 
de planejamento que conduz à ineficiência operacional, como relatado por Oloruntoba e Gray (2005). Contudo, a Organização Pan-Americana da Saúde (PAHO, 2000) contesta este raciocínio, afirmando que a maioria dos desastres, e as demandas por ajuda resultantes, são geralmente previsíveis e, portanto, ao estudar os programas de assistência humanitária anteriores, previsões mais precisas podem ser feitas, permitindo que regiões em risco se preparem e que as agências de socorro planejem os seus esforços. Van Wassenhove (2006) e PAHO (2000) compartilham a ideia de que a utilização do improviso como um elemento da resposta imediata ao desastre é um erro, que deve ser eliminado.

Para Beamon e Balcik (2008), abordagens pré-desastres que envolvem pré-posicionamento de estoques aumentam a capacidade das organizações de ajuda humanitária para mobilizar recursos e entregar suprimentos de ajuda rapidamente; embora possam ser financeiramente proibitivas. Assim, apenas algumas poucas organizações humanitárias podem suportar a despesa para armazenar e distribuir suprimentos de emergência. Apesar dos eventuais problemas que envolvem a aplicação de soluções em antecipação aos eventos naturais, estas proporcionam condições para a redução do esforço nas operações de resposta. As estratégias de preparação não se restringem ao pré-posicionamento de estoques, abrangendo também: o desenho da rede de suprimentos, o planejamento de estoques e problemas de localização utilizando ou não a infraestrutura existente; que proporcionam alternativas para o fluxo de materiais em cenários de desastres. O trabalho de Falasca et al. (2009) foi o único encontrado na pesquisa a tratar preventivamente da organização e do planejamento do recurso-humano voltado para o melhor desempenho operacional.

As soluções baseadas na etapa de resposta, em geral, visam atender às necessidades que surgem, atuando sobre o tempo de espera por socorro, a administração da demanda variável e a distribuição equitativa dos recursos normalmente escassos. A alternativa por modelos que integram as duas estratégias pode trazer benefícios para o processo como um todo ao avaliar previamente cenários e possíveis respostas, reduzindo o escopo de decisão quando da ocorrência do desastre. Entretanto, a dependência a dados de qualidade determinará o erro deste procedimento.

\subsection{Variáveis de decisão}

Para Beamon e Kotleba (2006), Beamon e Balcik (2008), Van Wassenhove (2006), Falasca et al. (2009) e Nagurney et al.(2012), o principal objetivo das organizações em atividades de resposta a desastres consiste na minimização do sofrimento por parte dos beneficiários. O Código de Conduta da Federação Internacional da Cruz Vermelha estabelece que a ajuda em operações de resposta a desastres deva ser distribuída com base na necessidade das vítimas (IFRC, 2009). Assim, para o desenvolvimento de modelos que visam otimizar operações no contexto humanitário, é fundamental a definição das características a serem consideradas, e como implementálas, para que se obtenham respostas de valor para as vítimas.

A avaliação dos artigos indicou três abordagens para a valorização dos objetivos humanitários nas respostas a desastres: (i) a aplicação de pesos a componentes de custos da função objetivo, induzindo a prioridade pelo impacto na minimização da função; (ii) a utilização de fatores de penalização em custos pelo não atendimento à demanda; e (iii) a baseada no estabelecimento de restrições que levem à equidade na distribuição entre os pontos demandantes.

Segundo Perez et al. (2010), os modelos que adotam a primeira abordagem apresentam técnicas que de alguma forma priorizam suas preocupações com as vítimas, relacionando-as aos custos operacionais através de medidas indiretas, entregando ao decisor a responsabilidade de determinar as prioridades. $\mathrm{O}$ resultado depende da experiência do profissional para estabelecer de forma adequada o critério de atendimento (considerações subjetivas) e a habilidade para readequar as ações de acordo com perfis específicos das áreas demandantes e as variações com o tempo.

Por sua vez, as linhas de desenvolvimento para gerar resultados compatíveis às expectativas no contexto humanitário através da minimização da demanda não atendida [Nagurney et al. (2012), Salmeron e Apte (2010), Clark e Culkin (2007), Beamon e Kotleba (2006)], ou da imposição de restrições de capital para garantir uma frequência mínima de entregas/distribuição dos recursos justa [Mete e Zabinsky (2009), Beamon e Balcik (2008), Clark e Culkin (2007) e Yushimito e Ukkusuri (2007)] são consideradas por Perez et al. (2010) como tentativas que podem 
causar eventuais problemas se impuserem níveis de serviço à ação de resposta que sejam inviáveis nas circunstâncias que se seguirem ao evento.

Perez et al. (2010) consideram que os custos logísticos se tornam uma preocupação secundária em ações humanitárias; e defendem a quantificação e incorporação de um componente que represente o sofrimento das vítimas a ser minimizado nos modelos. Contudo, McGuire (2001) e Van Wassenhove (2006) consideram que a gestão dos custos operacionais tem grande importância para o sucesso das ações de ajuda humanitárias devido ao aumento da responsabilidade das organizações não-governamentais perante seus doadores sobre a destinação dos fundos e à queda no volume de contribuições de financiamento, obrigando-as a planejar programas mais eficientes em custos.

$\mathrm{Na}$ análise dos artigos estudados, foram identificadas as variáveis de decisão em seus modelos, agrupando-os nas seguintes categorias: (i) demanda, voltados para a redução das necessidades não atendidas; (ii) atendimento, associados ao nível de serviço e à igualdade na distribuição da ajuda; (iii) tempos de resposta e de viagem, referente ao ciclo total de atendimento e ao da atividade de entrega; (iv) custos operacionais; e (v) motivação dos voluntários, medida indireta que diz respeito à alocação adequada de pessoal às atividades da logística humanitária, na busca de maior efetividade operacional. A Tabela 3 apresenta uma visão geral do momento para aplicação (pré ou pós-desastre) e das variáveis de decisão consideradas nos modelos estudados.

\section{APLICAÇÕES DA PESQUISA OPERA- CIONAL NO DESENVOLVIMENTO DOS MODELOS PROPOSTOS PELOS ARTIGOS SELECIONADOS}

No estudo dos artigos, identificou-se que as principais técnicas de Pesquisa Operacional (PO) adotadas nos modelos propostos são: programação linear (PL), estocástica (de dois estágios), inteira (PI), não linear (PNL) e de problemas de localização de instalações. Alguns autores oferecem aplicações integradas de técnicas, como Yi e Özdamar (2007), para a localização prévia de instalações integrando os problemas de roteirização e de localização; ou Özdamar et al. (2004) que propõem um modelo híbrido dos problemas de fluxo de rede multiproduto (PL) e de roteirização de veículos (PI) para a coordenação do processo de distribuição.

A definição dos níveis de estoque de itens de ajuda humanitária a serem mantidos para o rápido atendimento em resposta a desastres estão associados, em sua maioria, às formulações clássicas de gestão de estoques, como a aplicação de Emmett e Lodree (2011) do critério de decisão minimax (que otimiza o pior cenário) ao modelo do Lote Econômico de Pedido; a proposição de Beamon e Kotleba (2006) como extensão da política de estoque padrão com níveis de reposição para tamanhos de lotes; ou o modelo estocástico de estoque de Wyk et al. (2011). A aplicação diferenciada é de Ozbay e Ozguven (2007) que não utilizam formulação clássica, propondo um modelo estocástico não linear convexo, utilizando uma versão do Modelo Húngaro de Controle de Estoque (Prékopa, 2006).

A programação estocástica de dois estágios é preferencialmente utilizada em modelos que propõem decisões pré-desastre, com reavaliação após sua ocorrência, atualizando as condições das áreas atingidas e, em determinados trabalhos, com a indisponibilidade de alguns componentes da infraestrutura inicial. Salmeron e Apte (2010) aplicam esta técnica em um modelo de programação inteira mista para a otimização da cadeia de suprimentos em ações de ajuda humanitária. Da mesma forma, o trabalho de pré-posicionamento de estoques de Rawls e Turnquist (2009) utiliza o método L-shaped e um relaxamento Lagrangeano na solução. A proposta Mete e Zabinsky (2009) integra um problema de roteirização de veículos com capacidade reduzida. Barbarosoglu e Arda (2004) e Günnec e Salman (2007) desenvolvem aplicações de programação estocástica de dois estágios em modelos multicritério para roteirização de entregas e para o problema de localização de instalações na estruturação da rede logística humanitária.

O uso de programação linear foi mais característico nas propostas de otimização da estrutura da cadeia de suprimentos, como formulado por Nagurney et al. (2012) em um problema de desigualdade variacional (resolvido com a aplicação do método de Euller e de multiplicadores de Lagrange); ou no modelo multiproduto e multiobjetivo de Clark e Culkin (2007). 
Tabela 3 - Momento de aplicação e diretrizes de otimização dos modelos estudados

\begin{tabular}{|c|c|c|c|c|c|c|c|c|c|}
\hline & \multicolumn{5}{|c|}{$\begin{array}{l}\text { Atributos das variáveis de decisão dos } \\
\text { modelos }\end{array}$} & \multicolumn{2}{|c|}{$\begin{array}{c}\text { Momento do } \\
\text { desastre }\end{array}$} \\
\hline & & & 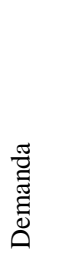 & 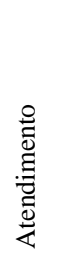 & 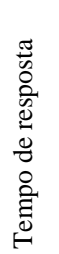 & 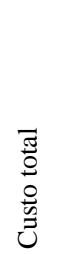 & 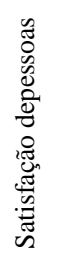 & 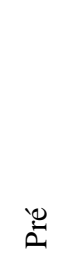 & $\ddot{2}$ \\
\hline \multirow{2}{*}{ 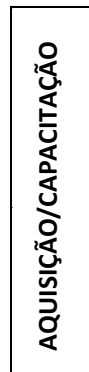 } & $\begin{array}{l}\text { Otimização da rede } \\
\text { de suprimentos }\end{array}$ & $\begin{array}{l}\text { Nagurney et al. (2012) } \\
\text { Nagurney et al. (2010) } \\
\text { Salmeron e Apte (2010) } \\
\text { Clark e Culkin (2007) } \\
\text { Beamon e Balcik (2005) }\end{array}$ & $\begin{array}{l}\square \\
\square \\
\square \\
\square \\
\square\end{array}$ & $\begin{array}{l}\square \\
\square \\
\square \\
\square \\
\square\end{array}$ & $\begin{array}{l}\square \\
\square \\
\square \\
\square \\
\square\end{array}$ & $\begin{array}{l}\square \\
\square \\
\square \\
\square \\
\square\end{array}$ & $\begin{array}{l}\square \\
\square \\
\square \\
\square \\
\square\end{array}$ & $\begin{array}{l}\square \\
\square \\
\square \\
\square \\
\square\end{array}$ & $\begin{array}{l} \\
\square \\
\square \\
\square \\
\square \\
\square\end{array}$ \\
\hline & $\begin{array}{l}\text { Pré-posicionamento } \\
\text { de produtos }\end{array}$ & $\begin{array}{l}\text { Rawls e Turnquist (2009) } \\
\text { Mete e Zabinsky (2009) } \\
\text { Yushimito e Ukkusuri (2007) } \\
\text { Beamon e Balcik (2008) }\end{array}$ & $\begin{array}{l}\square \\
\square \\
\square \\
\square\end{array}$ & $\begin{array}{l}\square \\
\square \\
\square \\
\square\end{array}$ & $\begin{array}{l}\square \\
\square \\
\square \\
\square\end{array}$ & $\begin{array}{l}\square \\
\square \\
\square \\
\square\end{array}$ & $\begin{array}{l}\square \\
\square \\
\square \\
\square\end{array}$ & $\begin{array}{l}\square \\
\square \\
\square \\
\square\end{array}$ & $\begin{array}{l}\square \\
\square \\
\square \\
\square\end{array}$ \\
\hline \multirow{3}{*}{ 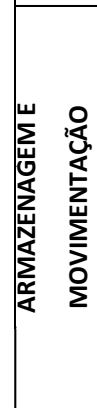 } & $\begin{array}{l}\text { Planejamento de } \\
\text { estoques }\end{array}$ & $\begin{array}{l}\text { Wyk et al. (2011) } \\
\text { Emmet e Lodree (2011) } \\
\text { Ozbay e Ozguven (2007) } \\
\text { Beamon e Kotleba (2006) }\end{array}$ & $\begin{array}{l}\square \\
\square \\
\square \\
\square\end{array}$ & $\begin{array}{l}\square \\
\square \\
\square \\
\square\end{array}$ & $\begin{array}{l}\square \\
\square \\
\square \\
\square\end{array}$ & $\begin{array}{l}\square \\
\square \\
\square \\
\square\end{array}$ & $\begin{array}{l}\square \\
\square \\
\square \\
\square\end{array}$ & $\begin{array}{l}\square \\
\square \\
\square \\
\square\end{array}$ & $\begin{array}{l}\square \\
\square \\
\square \\
\square\end{array}$ \\
\hline & $\begin{array}{l}\text { Localização de } \\
\text { instalações }\end{array}$ & $\begin{array}{l}\text { Jaller e Holguín-Veras (2011) } \\
\text { Günnec e Salman (2007) } \\
\text { Yi e Özdamar (2007) } \\
\text { Huang et al. (2012) }\end{array}$ & $\begin{array}{l}\square \\
\square \\
\square \\
\square \\
\end{array}$ & $\begin{array}{l}\square \\
\square \\
\square \\
\square\end{array}$ & $\begin{array}{l}\square \\
\square \\
\square \\
\square\end{array}$ & $\begin{array}{l}\square \\
\square \\
\square \\
\square \\
\square \\
\end{array}$ & $\begin{array}{l}\square \\
\square \\
\square \\
\square\end{array}$ & $\begin{array}{l}\square \\
\square \\
\square \\
\square\end{array}$ & $\begin{array}{l}\square \\
\square \\
\square \\
\square \\
\end{array}$ \\
\hline & $\begin{array}{l}\text { Alocação de } \\
\text { pessoal }\end{array}$ & Falasca et al. (2009) & $\square$ & $\square$ & $\square$ & $\square$ & $\square$ & $\nabla$ & $\square$ \\
\hline \multirow{2}{*}{ 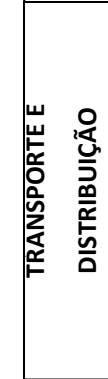 } & $\begin{array}{l}\text { Roteirização de } \\
\text { entregas }\end{array}$ & $\begin{array}{l}\text { Lin } \text { et al. }(2010) \\
\text { Perez } \text { et al. }(2010) \\
\text { Lin } \text { et al. }(2009) \\
\text { Campbell } \text { et al. (2008) } \\
\text { Barbarosoglu e Arda (2004) }\end{array}$ & $\begin{array}{l}\square \\
\square \\
\square \\
\square \\
\square\end{array}$ & $\begin{array}{l}\square \\
\square \\
\square \\
\square \\
\square\end{array}$ & $\begin{array}{l}\square \\
\square \\
\square \\
\square \\
\square\end{array}$ & $\begin{array}{l}\square \\
\square \\
\square \\
\square \\
\square\end{array}$ & $\begin{array}{l}\square \\
\square \\
\square \\
\square \\
\square\end{array}$ & $\begin{array}{l}\square \\
\square \\
\square \\
\square \\
\square\end{array}$ & $\begin{array}{l}\square \\
\square \\
\square \\
\square \\
\square\end{array}$ \\
\hline & $\begin{array}{l}\text { Coordenação do } \\
\text { fluxo de } \\
\text { distribuição }\end{array}$ & Özdamar et al. (2004) & $\square$ & $\square$ & $\square$ & $\square$ & $\square$ & $\square$ & $\square$ \\
\hline
\end{tabular}

A programação inteira tem aplicação também na roteirização de entregas, na localização de instalações e no problema de alocação de pessoal, apresentados, respectivamente, no desenvolvimento multiobjetivo de Lin et al. (2009 e 2010) - com solução por heurística de decomposição e atribuição; pela abordagem não linear de Jaller e Holguín-Veras (2011); e no modelo de otimização multicritério de Falasca et al. (2009) resolvido pelo método da fronteira eficiente (uso de uma curva de "trade-off" entre dois objetivos conflitantes). Yushimito e Ukkusuri (2007) combinam a programação inteira com a análise do caminho mais confiável, baseado na probabilidade de falha das rotas de transportes existentes. Ainda no campo da PI, a proposta de Perez et al. (2010) desenvolve o problema de roteiri- zação de veículos incluindo componentes sociais de aspecto humanitário, porém o modelo é de solução complexa e para aplicação em pequenos eventos.

Ao contrário de propor uma solução, o trabalho de Campbell et al. (2008) propõe uma discussão inserindo algoritmos do tipo minimax (minimização do tempo máximo de entrega) e minisum (minimização da soma dos tempos de entrega) como alternativas aos problemas convencionais do Caixeiro Viajante e de Roteirização de Veículos, verificando a equidade e a rapidez das soluções, que são as métricas do contexto humanitário escolhidas pelos autores. Beamon e Balcik (2005) utilizam o método clássico para problemas de localização de instalações para a minimização dos tempos de resposta 
em situações de emergência de pequena escala; e em 2008 os autores apresentaram uma variante do método da máxima cobertura para a localização de centros de distribuição e pré-posicionamento de estoques para uma resposta humanitária de âmbito global.

\section{ADEQUAÇÃO DOS MODELOS MATEMÁTICOS ÀS CONDIÇÕES OPERACIONAIS}

Esta seção aborda aspectos associados às características que cercam o desenvolvimento e a funcionalidade de cada modelo, no que diz respeito às possibilidades da sua aplicação nas condições operacionais da ação de resposta humanitária para a obtenção de melhores resultados. Para esta avaliação, foram considerados os seguintes fatores de adequação às condições operacionais reais das ações de resposta humanitária: (i) informações necessárias para a aplicação do modelo; (ii) grau de complexidade para a utilização do modelo; (iii) adequação ao ambiente operacional; e (iv) vinculação a organizações de ajuda humanitária.

\subsection{Informações necessárias para a aplicação do modelo}

É de fundamental importância a disponibilidade de dados para testes e aplicações de modelos matemáticos. Estes dados devem traduzir condições referentes à população, distâncias, tempos, infraestrutura e históricos de desastres ocorridos; de forma a possibilitar a montagem de cenários (local, danos materiais, mortes, impactados) com objetivo de preparação (etapa pré-desastre) voltada à redução do tempo de resposta e melhoria da disponibilidade de produtos, ou para o processo decisório de distribuição de bens e recursos durante a resposta efetiva pósdesastre. De Ville de Goyet e Morinière (2006) afirmam que o problema sofrido na Indonésia, em 2006, foi acentuado devido ao uso de dados de baixa qualidade coletados de fontes nacionais e locais, principalmente na validação, compilação e disseminação destes dados, impedindo que os decisores tivessem uma visão macro das necessidades.

Dos 25 artigos estudados, apenas nove tratam exclusivamente de aplicações pós-desastre, o que levou a maioria dos autores a estruturarem seus modelos baseados em áreas onde é identificada uma frequência regular de ocorrência de desastres naturais, não havendo uma preocupação quanto à disponibilidade e acesso aos dados; podendo utilizar o histórico dos acontecimentos como base para simulações de possíveis cenários pós-desastres (Özdamar et al., 2004; Clark e Culkin, 2007; Beamon e Kotleba, 2006). É possível, no entanto, avaliar e utilizar os dados mais importantes apresentados para estruturar as decisões de preparação aos desastres em regiões que tenham características cíclicas de ocorrências, ou mesmo as que tenham sofrido danos com desastres preparando-se para um novo acidente.

Dentre os nove modelos voltados exclusivamente para ações pós-desastre, não foi identificada a preocupação dos autores com a simplicidade e facilidade de captação dos dados para a aplicação dos modelos, e sim com a rapidez no seu processamento (Nagurney et al., 2010; Campbell et al., 2008), ou o uso de uma ferramenta mais simples (Beamon e Balcik, 2005). Como exemplo, os dados sobre custos, de grande utilização nos modelos, inclusive como variáveis para a aplicação de fatores associados a questões no contexto humanitário das operações, não têm uma clara definição sobre suas estruturações matemáticas, comprometendo a aplicação das propostas em um ambiente pressionado por condições de incerteza e urgência (Tomasini e Van Wassenhove, 2009).

\subsection{Grau de complexidade para a utilização do modelo}

Quanto mais tentam reproduzir as condições do ambiente de resposta a desastres naturais, mais os modelos se tornam complexos. Os modelos estudados, em sua maioria, buscam em seus desenvolvimentos a melhor representação das dificuldades enfrentadas, mas não apresentam suas estruturas de implantação, o que impede uma avaliação objetiva de quão complexo é utilizá-los. Entretanto, é possível estimar tal dificuldade com base na dependência de informações e critérios deixados a responsabilidade dos decisores para que possam ser executados. Assim, a complexidade dos modelos estudados está na utilização de pesos para definição de prioridades (Rawls e Turnquist, 2009; Barbarosoglu e Arda 2004; Mete e Zabinsky, 2009; Clark e Culkin, 2007), no conhecimento da confiabilidade do sistema de transportes (Yushimito e Ukkusuri, 2007), do risco de possíveis áreas afetadas por desastres (Beamon e Balcik, 2005) 
e no valor da vida humana (Wyk et al., 2011).

Entende-se que a complexidade no uso das propostas está além do conhecimento e experiência do decisor, mas também na utilização de pessoas do local na formação da equipe de operações, pois, embora não sejam capacitados em logística, detêm a experiência e o conhecimento tácito da região que permitirão o uso adequado dos modelos.

\subsection{Adequação ao ambiente operacional no contexto humanitário}

Uma das principais dificuldades encontradas no processo de modelagem de ações da logística humanitária consiste em representar por meio da função objetivo os principais valores que norteiam as operações de resposta a desastres, que são: senso de emergência, equidade na distribuição de suprimentos e redução do sofrimento das vítimas (Thomas e Mizushima, 2005). Assim, o tempo e o nível de atendimento à demanda são os fatores mais utilizados como variáveis de decisão na função objetivo com intuito de representar tais valores. Penalidades em custos associadas ao sacrifício e perdas de vítimas em função do nível de atendimento prestado também têm sido utilizadas.

A questão da demanda necessita de informações sobre os itens e as quantidades a serem supridas às vítimas. Em relação ao sofrimento das vítimas e perda de vidas, parâmetros financeiros alternativos foram apresentados por Perez et al. (2010), Jaller e Holguín-Veras (2011) e Wyk et al. (2011), com base em dados do Departamento de Transporte da África do Sul para a avaliação de acessibilidade e privação.

Autores como Rawls e Turnquist (2009), Barbarosoglu e Arda (2004), Mete e Zabinsky (2009) e Clark e Culkin (2007) utilizam pesos sobre os diversos componentes do custo logístico de acordo com a experiência dos responsáveis pelo processo decisório, com o intuito de priorizar a alocação de recursos. Entretanto, segundo Perez et al. (2010), há limitações importantes neste método, pois, por terem seu foco nestes objetivos, não são efetivos na busca da alocação de recursos que possibilite o maior benefício ao sistema. Os autores também fazem restrições aos métodos que minimizam a demanda não atendida (Beamon e Kotleba, 2006; Günnec e Salman, 2007; Yi e Özdamar, 2007; Lin et al., 2010) à busca de equidade na distribuição da ajuda humanitária, por desconsidera- rem as características de urgência individuais das demandas.

De acordo com a análise dos artigos selecionados, foi possível verificar que ainda não há uma definição quanto à melhor forma de representar os principais valores da logística humanitária na função objetivo dos modelos matemáticos. No entanto, é importante ressaltar que, para todas alternativas apresentadas como forma de abordar o problema, deve-se manter um procedimento de controle e reavaliação capaz de readequar as decisões às mudanças das condições operacionais ao longo do tempo.

\subsection{Vinculação a organizações ou ações de ajuda humanitária}

Trata-se de um fator importante que facilita a obtenção de dados e permite agregar o conhecimento e experiência de pessoas que participam de operações humanitárias e conhecem efetivamente os problemas e as reais condições de campo ao desenvolvimento de modelos matemáticos. Trabalhos como os de Beamon e Balcik (2008), Wyk et al. (2011), Mete e Zabinsky (2009), Beamon e Kotleba (2006) e Özdamar et al. (2004), que contam com o apoio ou participam de programas voltados para o desenvolvimento de soluções de otimização de ações logísticas no contexto humanitário, têm como vantagens o apoio para a obtenção de recursos e dados, além da aplicação em condições reais. Esta vantagem subjetiva está no fato de seus patrocinadores esperam por resultados efetivos que venham a ser aplicados em suas operações de ajuda humanitária. Da mesma forma, espera-se que a disponibilidade de dados sobre acidentes e a experiência operacional destas organizações possibilitem aos pesquisadores o acesso a informações do campo que tornem a formulação de seus modelos mais completa.

\section{SUGESTÕES PARA A APLICAÇÃO DE SOLUÇÕES MATEMÁTICAS PARA A LOGÍSTICA HUMANITÁRIA NO CONTEXTO NACIONAL}

A maioria dos desastres naturais no país relacionam-se a situações de mudanças climáticas, como deslizamentos de terra, inundações e tempestades (Bertazzo et al., 2013). Um dos objetivos da presente pesquisa consiste em analisar a possibilidade de aplicações matemáticas para melhorar a resposta humanitária aos desastres no 
Brasil. Desta forma, três pontos importantes sobre o contexto brasileiro em termos de logística humanitária devem ser considerados:

- Escassez de dados e de detalhamento das regiões em condições suficientes para possibilitar uma modelagem matemática que possa ser validada nas condições típicas dos desastres no Brasil (Costa et al., 2012).

- A experiência adquirida nas respostas humanitárias aos desastres naturais ocorridos no país está concentrada na Defesa Civil, nas Forças Armadas e, de forma pessoal, nos voluntários que participaram das ações (Bandeira et al., 2011; Costa et al., 2012).

- Algumas regiões brasileiras têm características de ocorrência cíclica de desastres naturais e, a cada ano, os eventos ocorrem sem uma adequada melhoria na ação de resposta (Bandeira et al., 2011).

Baseado nestas premissas, é verificada a necessidade do desenvolvimento de modelos matemáticos com foco pré-desastre para aplicação em cenários desenvolvidos com base nas características dos desastres naturais ocorridos no Brasil. Tais modelos devem fazer parte de um projeto mais amplo que tenha como objetivo a estruturação da rede humanitária em operações de respostas a desastres, servindo como ferramenta de apoio à decisão para determinar as ações necessárias para a preparação nos períodos que precedem o ciclo de desastres. Assim, o uso de modelos matemáticos para a otimização dos resultados deve ser parte de um conjunto de ações que possibilitem ao país a melhoria da sua capacidade de resposta a desastres.

A logística humanitária ainda é um tema de pesquisa incipiente no Brasil. Bertazzo et al. (2013) destacam a importância do desenvolvimento de estudos e pesquisas sobre a temática em ambiente brasileiro. De acordo com os autores (2014), até 2013, havia somente um artigo sobre logística humanitária publicado em periódicos nacionais. Nicholson (2003), na Revista Transportes, analisa a confiabilidade das redes de transporte, técnicas de modelagem e técnicas de avaliação em caso de desastres naturais. Em 2013, Bertazzo et al. (2013) também publicaram na Revista Transportes uma revisão da literatura acadêmica brasileira sobre o tema. Atualmente, há grupos de pesquisadores estudando o assunto na Universidade Federal de Santa Catarina, na
Universidade de São Paulo, na Pontifícia Universidade Católica do Rio de Janeiro e no Instituto Militar de Engenharia. Contudo, a maioria dos trabalhos publicados ainda é em congressos. Segundo Bertazzo et al. (2013), até 2013, foram identificados vinte artigos publicados no congresso anual da ANPET (Associação Nacional de Pesquisa e Ensino em Transportes), seis no ENEGEP (Encontro Nacional de Engenharia de Produção) e um artigo no ENANPAD (Encontro Nacional da Associação Nacional de Pós-graduação e Pesquisa em Administração). Desta forma, o país tem potencial acadêmico capaz de produzir em conjunto com os governos, da Defesa Civil e das Forças Armadas (os principais atores nas ações de resposta humanitária) soluções para os problemas antes mencionados. Assim, a visão de uma cadeia de suprimentos humanitária integrada permitirá resultados mais eficazes, levando à redução dos custos de resíduos e operacional, com o consequente aumento do nível de serviço oferecido às vítimas (onde a aplicação de técnicas de pesquisa operacional será essencial).

\section{CONCLUSÕES}

Segundo Forrester (1971), existe uma tendência à utilização de modelos baseados na experiência e na intuição, mesmo em situações complexas, quando a relação de causa e efeito não é clara. Esta é a realidade encontrada em operações humanitárias em resposta a desastres naturais. No entanto, neste contexto, modelos deste tipo não são suficientes para contemplar toda a operação, considerando-se também o fato de que a intuição e a experiência humana são afetadas por fatores irracionais, como emoções e pressão do tempo. Ainda, o atraso na tomada de decisão tende a afetar, entre outras coisas, a eficiência das equipes de socorro em atender as necessidades dos vitimados no prazo necessário.

O estudo dos modelos matemáticos apresentados mostra que ainda há uma lacuna entre o que é modelado e o que realmente ocorre no campo em operações de resposta a desastres (no ambiente dos desastres). Existem importantes avanços na área, como a inclusão de aspectos humanitários por meio de variáveis de decisão na formulação destes modelos. Contudo, não se verifica a possibilidade de uma utilização direta dos modelos propostos no processo de resposta aos desastres, com a expectativa de que algum 
dos problemas identificados seja efetivamente solucionado ou tenha a perspectiva de solução.

O levantamento e análise realizada neste artigo mostram que os modelos propostos parecem dispersos, com pouca sinergia entre as diferentes propostas e suas aplicações. Tais modelos oferecem alternativas, mas não complementares e, com algumas exceções, apresentam pouca aderência às condições de campo. Este é um dos principais pontos que precisam ser melhorados para motivar os gerentes de operações a usar modelos matemáticos propostos.

\section{REFERÊNCIAS}

BANDEIRA, R. A.M, CAMPOS, V.B.G, e BANDEIRA, A.P. F. (2011) Uma visão da logística de atendimento à população atingida por desastre natural. Anais do XXV Congresso de Pesquisa e Ensino em Transportes, ANPET, Belo Horizonte.

BARBAROSOGLU, G e ARDA, Y (2004) A two-stage stochastic programming framework for transportation planning in disaster response. Journal of the Operational Research Society (2004) 55, p. 43-53. DOI:10.1057/palgrave.jors.2601652.

BEAMON, B. M. e BALCIK, B. (2005) Distribution Network Design for Humanitarian Relief Chains. IE Graduate Seminar, November 8, 2005 IE Graduate Seminar, November 8, 2005. http://courses.washington.edu/ie59x/abstracts/IEseminar05.pdf

BEAMON, B. M. e BALCIK, B. (2008) Facility location in humanitarian relief. International Journal of Logistics: Research and Applications. Vol. 11, No. 2, April 2008, 101-121.

BEAMON, B. M. e KOTLEBA, S. A. (2006) Inventory modeling for complex emergencies in humanitarian relief operations. International Journal of Logistics: Research and Applications, Vol. 9, No. 1, 2006, pp. 1-18. DOI: $\underline{10.1080 / 13675560500453667 .}$

BERTAZZO, T., BRITO JUNIOR, I., LEIRAS, A. e YOSHIZAKI,H. (2013) Revisão da literatura acadêmica brasileira sobre a gestão de operações em desastres naturais com ênfase em logística humanitária. Transportes, v. 21, n.3, p. 31-39. DOI:10.4237/transportes.v21i3.633.

CAMPBELL, A. M. VANDENBUSSCHE, D. HERMANN, W. (2008) Routing for Relief Efforts. Transportation Science 20085 Volume: 42 Issue Number: 2 pp 127-145 Publisher: Institute for Operations Research and the Management Sciences. DOI: $\underline{10.1287 / \text { trsc. } 1070.0209 \text {. }}$

CASTRO, Antônio Luiz Coimbra de. Manual de planejamento em defesa civil. Vol.1. Brasília: Imprensa Nacional, 1999.

CLARK, A. e CULKIN, B.(2007) A Network Transshipment Model for Planning Humanitarian Relief Operations after a Natural Disaster. In: 22nd European Conference on Operational Research, vol. 44; 2007. p. 1e34.

COSTA, S.R.A, BANDEIRA, R.A.M, CAMPOS, V.B.G (2012) Uma análise do processo de distribuição na cadeia de suprimentos me operações humanitárias. Anais do XXXII Encontro Nacional de Engenharia de Produção, ENEGEP, Bento Gonçalves.
DE BEER, E.J.H. e VAN NIEKERK, E. (2004)The estimation of unit costs of road traffic accidents in South Africa.Technical report, National Department of Transport.

DE VILLE DE GOYET, C. e MORINIÈRE, L.(2006) The role of needs assessment in the tsunami response. Tsunami Evaluation Coalition, London.

EMMETT, J. e LODREE, J. (2011) Pre-storm emergency supplies inventory planning. Journal of Humanitarian Logistics and Supply Chain Management Vol. 1 No. 1, 2011 pp. 50-77

FALASCA, M., ZOBEL, C. W. e FETTER, G. M. (2009) An optimization model for humanitarian relief volunteer management. Proceedings of the 6th International ISCRAM Conference - Gothenburg, Sweden, May 2009 J. Landgren and S. Jul, eds.

FORRESTER, J.W. (1971) Counterintuitive behavior of social systems. Technology Review, Vol. 73 No. 3, pp. 52-68. DOI: 10.1007/BF00148991.

GUHA-SAPIR, Debarati; HOYOIS, Philippe e BELOW, Regina.(2013) Annual Disaster Statistical Review 2012: The numbers and trends. Centre for Research on the Epidemiology of Disasters. Brussels: CRED; 2013. Disponível em: <http://cred.be/sites/default/files/ADSR_2012.pdf>. Acessoem: 13 nov 2013.

GUNNEC, D. e SALMAN, F. (2007) A two-stage multi-criteria stochastic programming model for location of emergency response and distribution centers. In: International Network Optimization Conference - INOC 2007. Acessado em 14/07/201 peloendereçohttp://www.mendeley.com/research/twostagemulticriteria-stochastic-programming-model-location-emergency-response-distribution-centers/

HUANG, M. SMILOWITZA, K. BALCIK, B. (2012) Models for relief routing: Equity, efficiency and efficacy. Transportation Research Part E: Logistics and Transportation Review Volume 48, Issue 1, January 2012, Pages 2-18 Select Papers from the 19th International Symposium on Transportation and Traffic Theory. DOI: 10.1016/j.sbspro.2011.04.525.

IFRC: International Federation of Red Cross and Red Crescent Societies (2000) Disaster Preparedness Training Programme. 2000. Disponível em: <http://www.ifrc.org/Global/Introdp.pdf $>$. Acesso em: 23 nov 2013.

IFRC: International Federation of Red Cross and Red Crescent Societies (2009) Emergency items catalogue. Available at http://procurement.ifrc.org/catalogue/ Acesso em: 23 nov 2013.

JALLER, M. e HOLGUÍN-VERAS, J .(2011) Locating Points of Distribution in Disasters with Social Costs Considerations. Submitted to the Transportation Research Board (TRB) 90th Annual Meeting, Washington, D.C. Acessado em 15/07/2012 no endereço: http://transp.rpi.edu/ HUM-LOG/Doc/PODs.pdf

KOVACS, G. e SPENS, K. (2007). Humanitarian logistics in disaster relief operations. International Journal of Physical Distribution \& Logistics Management, v. 37, n. 2, p. 99-114.

LIN, Y. H. BATTA, R. ROGERSON, P. A. BLATT, A. FLANIGAN, M. (2009) Application of a Humanitarian Relief Logistics Model to an Earthquake Disaster. Transportation Research Record http://www.citeulike.org/user/hmedal/article/9217419

LIN, Y.H., BATTA, R., ROGERSON, P.A., BLATT, A., FLANIGAN, M., LEE, K.(2010) A Logistics Model for Emergency Supply of Critical Items in the Aftermath of a Disaster. 
Socio-Economic Planning Sciences Volume 45, Issue 4, December 2011, Pages 132-145 http://www.acsu.buffalo.edu/ batta/trb Batta.pdf. DOI: 10.1016/j.seps.2011.04.003.

LONG, D. e WOOD, D. (1995) The Logistics of Famine Relief. Journal of Business Logistics. 16, 213-229.

McGUIRE, G.(2001) Supply Chain Management in the Context of International Humanitarian Assistance in Complex Emergencies - Part 2. Supply Chain Practice. 3, 4-18.

METE, H. O. e ZABINSKY, Z. B.(2009) Preparing for Disasters: Medical Supply Location and Distribution. Retrieved January, 252009. http://parvac.washington.edu/papers/downloadpaper.phd/id=13/parvac 13.pdf

NAGURNEY, A. YU, M. e QIANG, Q. (2010) Supply chain network design for critical needs with outsourcing. Papers in Regional Science, Volume 90 Number 1 March 2011.

NAGURNEY, A. YU, M. e QIANG, Q. (2012) Multiproduct Humanitarian Healthcare Supply Chains: A Network Modeling and Computational Framework. Proceedings of the 23rd Annual POMS Conference, Chicago, Illinois, April 20-23, 2012.

NATARAJARATHINAM, M.; CAPAR, I. E NARAYANAN, A. (2009) Managing supply chains in times of crisis: a review of literature and insights. International Journal of Physical Distribution and Logistics Management 39(7), 535-573.DOI: 10.1108/09600030910996251.

OLORUNTOBA, R. and GRAY, R. (2005) Humanitarian aid: an agile supply chain? Supply Chain Management. 11, 115-120. DOI: $10.1108 / 13598540610652492$.

OZBAY, K. e OZGUVEN, E.(2007) A Stochastic Humanitarian Inventory Control Model for Disaster Planning. Journal of the Transportation Research Board. Issue Number: 2022. pp 63-75 Publisher: Transportation Research Board ISSN: 0361-1981. DOI: $10.3141 / 2022-08$.

OZDAMAR, L. EKINCI, E. e KUCUKYAZICI. B. (2004) Emergency Logistics Planning in Natural Disasters. Annals of Operations Research. 129, 217-245.

PAN-AMERICAN HEALTH ORGANISATION (PAHO) (2000) Manual Logistical Management of Humanitarian Supply. 2000, www.disaster-info.net/SUMA/english/software/manuals/MISEManualEnglish.pdf

PEREZ, N. HOLGUIN-VERAS, J. MITCHELL, J.E. e SHARKEY, T.C. (2010) Integrated Vehicle Routing Problem with Explicit Consideration of Social Costs in Humanitarian Logistics. http://transp.rpi.edu/ HUM-LOG/Doc/IRP.pdf

PRÉKOPA, A. (2006) On the Hungarian Inventory Control Model. European Journal of Operational Research, Vol. 171, 2006, pp. 894-914. DOI: 10.1016/j.ejor.2005.01.008

RAWLS, C.G. TURNQUIST, M.A.(2009) Pre-positioning of emergency supplies for disaster response. Transportation Research Part B; 44:521e34.DOI: 10.1109/ISTAS.2006.4375894.

SALMERON, J. e APTE, A. (2010) Stochastic Optimization for Natural Disaster Asset Prepositioning. 574 Production and Operations Management 19(5), pp. 561-574.

SAMPSON, S. E.(2006) Optimization of volunteer labor assignments. Journal of Operations Management, 24, 4, 363-377. DOI: 10.1111/i.1937-5956.2009.01119.x.

THOMAS, A.S. e KOPCZAK, L.R. (2005) From Logistics to Supply Chain Management: The Path Forward in the Humanitarian Sector. Fritz Institute.

THOMAS, A. MIZUSHIMA, M.(2005) Logistics training: Necessity or luxury?Forced Mitig.Rev. 22: 60-61.

THOMAS, A.S. (2003) Humanitarian Logistics: Enabling Disaster Response. Fritz Institute.

TOMASINI, R. and VAN WASSENHOVE, L.V. (2009) $\mathrm{Hu}$ manitarian logistics. Palgrave Macmillan.

VAN WASSENHOVE, L. (2006) Humanitarian aid logistics: supply chain management in high gear. Journal of the Operational Research Society. 57, 475-498.DOI: 10.1057/palgrave.jors.2602125.

WYK, E. V. BEAN, W.L. e YADAVALLI, V.S.S.(2011) Modeling of uncertainty in minimizing the cost of inventory for disaster relief. South African Journal of Industrial Engineering May 2011 Vol. 22(1): 1-11.

YI, W. e OZDAMAR, L. (2007) A dynamic logistic coordination model for evacuation and support in disaster response activities. European Journal of Operational Research. 179, 1177-1193. DOI: 10.1016/j.ejor.2005.03.077.

YUSHIMITO, W. F. e UKKUSURI, S. V. (2007) Location routing approach for the humanitarian prepositioning problem. Transportation Research Record: Journal of the Transportation Research Board, Vol. 2089, No. -1. (2007), pp. 18-25. 